\title{
An application of PCR-RFLP species identification assay for environmental DNA detection
}

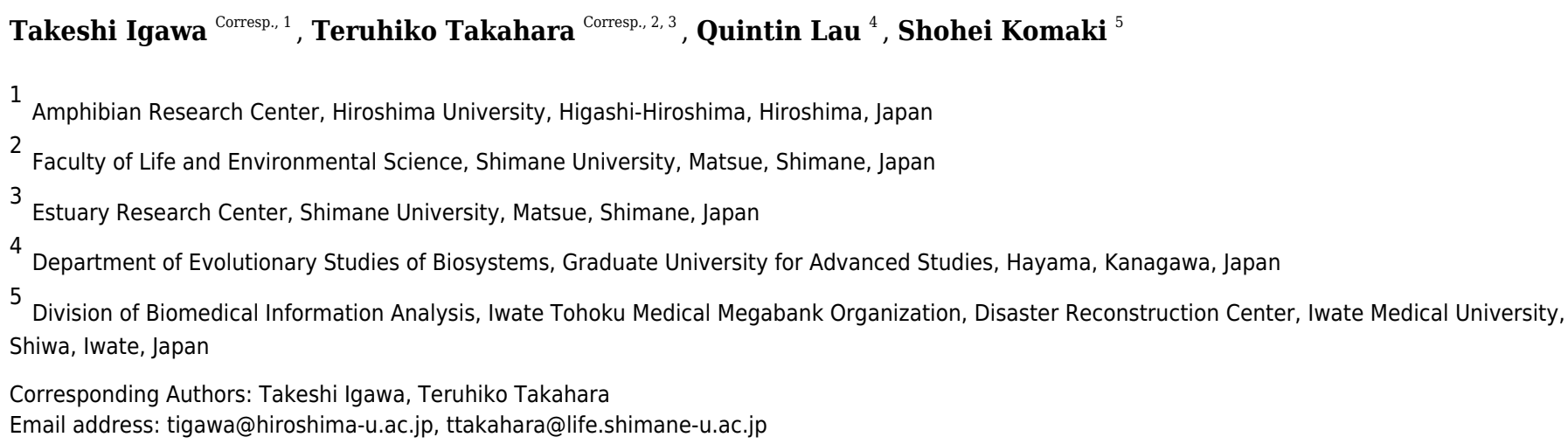

Recent advancement of eDNA methods for surveying species in aquatic ecosystems has been used for various organisms and contributed to monitoring and conservation of species and environments. Amphibians are one of the promising taxa which could be monitored efficiently by applying quantitative PCR or next generation sequencing to eDNA. However, the cost of eDNA detection using these approaches can be quite high and requires instruments that are not usually installed in ecology laboratories. For aiding researchers in starting eDNA studies of amphibians, especially those not specialized in molecular biology, we developed a cost efficient protocol using PCR-RFLP method. We attempted to detect eDNA of three Japanese Rana species ( $R$. japonica, $R$. ornativentris, and $R$. tagoi tagoi) in various spatial scales including an area close to the Fukushima Nuclear Power Plant (FNPP) where the environment is recovering after the disaster in 2011. Our PCR-RFLP protocol was successful in detecting Rana species in static water in both laboratory and field; however, it could not detect Rana species in non-static water samples from the field. Even a more sensitive detection method (standard qPCR) was unable to detect frogs in all non-static water samples. We speculate that our new protocol is effective for frogs living in lentic habitats, but not for lotic habitats which may still require the gold standard of field observation for detection approach. 
1 An application of PCR-RFLP species identification 2 assay for environmental DNA detection.

3

\author{
Takeshi Igawa $^{1}$, Teruhiko Takahara ${ }^{2,3}$, Quintin Lau ${ }^{4}$, Shohei Komaki ${ }^{5}$ \\ ${ }^{1}$ Amphibian Research Center, Hiroshima University, Higashi-Hiroshima, Hiroshima, Japan \\ ${ }^{2}$ Faculty of Life and Environmental Science, Shimane University, Matsue, Shimane, Japan \\ ${ }^{3}$ Estuary Research Center, Shimane University, Matsue, Shimane, Japan \\ ${ }^{4}$ Department of Evolutionary Studies of Biosystems, The Graduate University for Advanced \\ Studies, Hayama, Kanagawa, Japan \\ ${ }^{5}$ Division of Biomedical Information Analysis, Iwate Tohoku Medical Megabank Organization, \\ Disaster Reconstruction Center, Iwate Medical University, Shiwa, Iwate, Japan
}

Corresponding Author:

Takeshi Igawa $^{1}$, Teruhiko Takahara ${ }^{2}$

11-3-1 Kagamiyama, Higashi-Hiroshima, Hiroshima, 739-8526, Japan

${ }^{2} 1060$ Nishikawatsu-cho, Matsue, Shimane, 690-8504, Japan

Email address: tigawa@hiroshima-u.ac.jp (TI), ttakahara@life.shimane-u.ac.jp (TT)

\title{
Abstract
}

Recent advancement of eDNA methods for surveying species in aquatic ecosystems has been used for various organisms and contributed to monitoring and conservation of species and environments. Amphibians are one of the promising taxa which could be monitored efficiently by applying quantitative PCR or next generation sequencing to eDNA. However, the cost of eDNA detection using these approaches can be quite high and requires instruments that are not usually installed in ecology laboratories. For aiding researchers in starting eDNA studies of amphibians, especially those not specialized in molecular biology, we developed a cost efficient protocol using PCR-RFLP method. We attempted to detect eDNA of three Japanese Rana species ( $R$. japonica, $R$. ornativentris, and $R$. tagoi tagoi) in various spatial scales including an area close to the Fukushima Nuclear Power Plant (FNPP) where the environment is recovering after the disaster in 2011. Our PCR-RFLP protocol was successful in detecting Rana species in static water in both laboratory and field; however, it could not detect Rana species in non-static water samples from the field. Even a more sensitive detection method (standard qPCR) was unable to detect frogs in all non-static water samples. We speculate that our new protocol is effective for frogs living in lentic habitats, but not for lotic habitats which may still require the gold standard of field observation for detection approach. 
40

41

42

43

44

45

46

47

48

49

50

51

52

53

54

55

56

57

58

59

60

61

62

63

64

65

66

67

68

69

70

71

72

73

74

75

76

77

78

79

\section{Introduction}

Spatial distribution of organisms is one of the key components for understanding the ecology of the species and the ecosystem in which they inhabit. In addition, monitoring of species and population distribution are essential for their conservation. However, traditional visual surveys and monitoring techniques can be problematic, related to seasonal, ecological, and ethological differences within a target species, and difficulties in distinguishing between closely related sympatric species and varied life stages. In recent studies, environmental DNA (eDNA) methods, which involve the detection of species-specific DNA fragments in the environment, have been used for surveying species in aquatic ecosystems (Takahara, Minamoto \& Doi, 2013; Rees et al., 2014; Goldberg, Strickler \& Pilliod, 2015). Although there are some issues related to field surveys, this is a promising and non-invasive method, and thus has been used for various organisms including both plants and animals in terrestrial and aquatic environments (Thomsen \& Willerslev, 2015).

However, such eDNA detection experiments usually require expensive instruments and reagents for quantitative PCR (qPCR) (for single species) or next generation sequencing (NGS) (for multiple species). This potentially limits the number of institutions able to conduct eDNA studies. For sustainable monitoring activities and conservation of species diversity, an efficient and easy method should be developed for many laboratories close to a field site. Especially for amphibians, several studies support that detection is more cost-effective with eDNA than with traditional monitoring approaches (Olson, Briggler \& Williams, 2013; Pilliod et al., 2013; Biggs et al., 2015; Buxton et al., 2017). Amphibians are also intrinsic target species for eDNA studies in terms of their ecology because they have both aquatic and terrestrial life cycles and inhabit different kinds of water bodies, such as river (lotic) and pond (lentic) water systems, depending on species and life stages.

Although the sensitivity and cost of detection methods could be correlated, applying the low cost PCR-RFLP for eDNA detection has already been successful in differentiating several salmonid fish species (Clusa et al., 2017). However, to-date this PCR-RFLP approach has not been applied to organisms that are not fully aquatic, including amphibians. Therefore, it would be important to develop a cost efficient protocol using PCR-RFLP method for detection of eDNA fragments, instead of qPCR, in amphibian species.

In this study, we attempted to develop a protocol for detecting the presence of three true frog species from the Rana genus ( $R$. japonica, $R$. ornativentris and $R$. tagoi tagoi). These species are distributed across mainland Japan, including Honshu, Shikoku, and Kyushu islands. Rana japonica and $R$. ornativentris are often located near areas of human development and thus could be threatened with population decline due to human-related habitat loss. As a result, these two species have been listed as locally threatened in some regions (e.g. Natural Environment Division, Bureau of Environment, 2010; Natural Environment Division, Depertment of Environmental Affairs, 2014). In addition, these species are key indicator species for assessing the degree of disturbance to the natural environment, especially in Japanese traditional rural 
80

81

82

83

84

85

86

87

88

89

90

91

92

93

94

95

96

97

98

99

100

101

102

103

104

105

106

107

108

109

110

111

112

113

114

115

116

117

118

119

areas called "Satoyama" (Kitagawa, 2002). For example, agencies like the Biodiversity Center of Japan, the Nature Conservation Bureau, and the Ministry of the Environment (e.g. MonitoringSite 1000 project) have conducted ecological monitoring surveys and listed the occurrence of Rana species in numerous reports. Habitat selection of the three species differs to some extent, whereby each species inhabits a wide range of microhabitats. Rana japonica and $R$. ornativentris are mainly found in grasslands that range from lowlands to hillsides; they migrate to marshes, ponds, or paddy fields before the breeding season in winter (Maeda \& Matsui, 1999), but $R$. ornativentris tends to dwell at higher elevations than $R$. japonica (Kuramoto \& Ishikawa, 2000; Osawa \& Katsuno, 2001). Another brown frog species, $R$. tagoi tagoi, resembles $R$. ornativentris in morphology and is distributed in hilly areas, sometimes in sympatry with the other two species. However, R. t. tagoi lay their eggs under the ground on the edges of small mountain streams during spring.

For development of our new protocol in this study, we adapted the PCR-RFLP method that recognizes these three Rana species (Igawa et al., 2015) for eDNA detection. In this method, mitochondrial 16S rRNA fragments were amplified and digested with two restriction enzymes (SpeI and HphI) to identify species within the samples by electrophoretic banding pattern of the digested fragments. To evaluate the performance of our protocol, we attempted to detect eDNA diluted in water samples and compared the results with visual observation and qPCR methods.

To test applicability in various spatial scales, various water samples were collected from regional levels to microhabitat in addition to water tank experiments. In particular, we obtained regional samples covering $60 \mathrm{~km}$ from Fukushima Nuclear Power Plant (FNPP) in spring and summer seasons and assessed presence information of $R$. t. tagoi in habitats recovering after the disaster in 2011. Finally, we reported the effectiveness of the protocol for distribution surveys in the field.

\section{Materials \& Methods}

\section{Water tank experiment}

To characterize basic performance of our PCR-RFLP assay for environmental DNA detection, we firstly conducted model experiments by preparing eight different water tank culture setups with the following animals: (1) forty $R$. japonica tadpoles for two days, (2) forty $R$. japonica tadpoles for five days, (3) nine $R$. japonica and one $R$. ornativentris adults for one day, (4) one $R$. japonica and nine $R$. ornativentris adults for one day, (5) five $R$. japonica and five $R$. ornativentris adults for one day, (6) one $R$. t. tagoi and nine $R$. japonica adults for one day, (7) one R. t. tagoi and nine R. ornativentris adults for one day, and (8) negative control (to check the specificity of our method)- no animals for one day [but water was used previously for other frog species (Rhacophorus arboreus)]. The tadpoles of R. japonica were collected from a pond in Hiroshima University campus in Higashi-Hiroshima city (Hiroshima Prefecture) in April 2016 just before the experiment and were classified as growth stage 33 according to Tahara (1974) [equivalent to stage 37 in Gosner (1960)]. The adult frogs of $R$. japonica, $R$. ornativentris, and $R$. 
120 t. tagoi were collected from Higashi-Hiroshima city in 2014, Kita-Hiroshima-cho (Hiroshima 121 Prefecture) in 2015, and Kisa, Miyoshi city (Hiroshima Prefecture) in 2011, respectively, and 122 reared in the Amphibian Research Center, Hiroshima University, until the start of experiments. 123 We transferred and kept animals in plastic tanks $(450 \times 650 \times 150 \mathrm{~mm})$ filled with $8 \mathrm{~L}$ aged tap 124 water (water level about $30 \mathrm{~mm}$ which immersed half body of adults). The animals were not fed 125 during the experiment and were held at room temperature around $18^{\circ} \mathrm{C}$ under $12 \mathrm{~h}: 12 \mathrm{~h} \mathrm{light-}$

126

127

128

129

130

131

132

133

134

135

136

137

138

139

140

141

142

143

144

145

146

147

148

149

150

151

152

153

154

155

156

157

158 dark cycle. We collected a $500 \mathrm{~mL}$ water sample from each tank after the specific periods according to the treatments using DNA-free $500 \mathrm{~mL}$ bottles, and samples were immediately transferred to a $-30^{\circ} \mathrm{C}$ freezer. These experiments were conducted in April 2016 for tadpoles (tanks 1 and 2) and September 2016 for adults (tanks 3 to 8); all animals were returned to the original habitat or enclosures under healthy conditions soon after the experiments.

All procedures were approved by the Hiroshima University Animal Research Committee (Approval number: G17-4) and were carried out in accordance with the recommendations in the Guide for the Care and Use of Laboratory Animals of the Hiroshima University Animal Research Committee.

\section{Field survey and experiments}

To check the performance and sensitivity of PCR-RFLP assay for eDNA from the field environment, we collected water samples from ponds and rivers at 28 sites (Fig. 1). For validation of applicability to monitoring studies in various spatial scales, we collected samples in three hierarchical levels: (A) regional samples covering $60 \mathrm{~km}$ from Fukushima Nuclear Power Plant (FNPP) (sample no. 1-20), (B) local area samples from a single body of water in Hiroshima University campus, Higashi-Hiroshima (sample no. 21 -26), and (C) microhabitat level samples from a single breeding site of R. japonica in Etajima Island, Hiroshima Prefecture (N 34.27067, E 132.47679) (sample no. 27 and 28). Following to Takahara et al. (in press), we took $500 \mathrm{ml}$ by filling sodium hypochlorite-treated $500 \mathrm{ml}$ bottles (e.g. DNA-free bottles). Field survey was conducted in spring and summer because the true Rana species are active in these seasons. Samples from Fukushima (A) were collected during two periods, 12 to 14 May 2015 and 11 to 13 August 2015. Samples from Hiroshima University (B) and Etajima (C) were collected on 11 April and 17 April in 2016, respectively. To prevent contamination by a field sampler, we began collecting at downstream sites, and moved upstream as subsequent samples were collected. Sampled water bottles were transported on ice in a cooling box to the laboratory and stored at $-30^{\circ} \mathrm{C}$. The subsequent procedure of filtering water samples that were stored frozen was performed within three weeks.

In addition, we recorded the presence and absence of frogs and/or tadpoles by visual observation, exploring the vicinity of the field sampling sites. Basically, three or four people conduct field observation for more than 20 min at each site. For sampling in Fukushima, permission to enter the survey area was obtained from the local governments [Iitate Vilage (approval number: 2705-01 and 2707-0) and Namine Town (approval number: 755 and 1959)]. 
159

160

161

162

163

164

165

166

167

168

169

170

171

172

173

174

175

176

177

178

179

180

181

182

183

184

185

186

187

188

189

190

191

192

193

194

195

196

197

198

Notification of the field survey was accepted by the Iwaki District Forest Office, and permits were obtained from private landowners as required for each site.

\section{DNA extraction and PCRs}

To avoid contamination, we followed a unidirectional lab flow, whereby we performed all PCR protocols, including preparation/addition of the standards and qPCR cycling, in two separate rooms (rooms 1 and 2, respectively). To prevent carry-over contamination, no equipment or samples were returned from room 2 to room 1 . In both rooms, laboratory benches were decontaminated using commercial bleach.

The water samples stored at $-30^{\circ} \mathrm{C}$ were thawed and vacuum-filtered through glass microfiber filters (GF/F, GE Healthcare Bio-Sciences, Pittsburgh, PA, USA) with $0.7 \mu \mathrm{m}$ mesh size, which were used in other amphibian studies (e.g. Katano et al., 2017; Iwai, Yasumiba \& Takahara, 2019). Filter funnels and tweezers used in the filtration treatment were sterilized with $10 \%$ commercial bleach (ca. $0.6 \%$ hypochlorous acid) for $5 \mathrm{~min}$ (i.e. sodium hypochlorite treatment), flushed with a large amount of tap water, and then rinsed with DNA-free distilled water between samples to avoid cross contamination. Filtering controls (i.e. $500 \mathrm{~mL}$ of distilled water) in laboratory experiment controls filtered on each day of sample filtration. The filter papers were wrapped in new aluminum foil (i.e. DNA-free), placed in plastic bags, and stored at $-30^{\circ} \mathrm{C}$. The eDNA was extracted from the filters according to the methods of Uchii, Doi \& Minamoto (2016), using a Salivette tube (Sarstedt, Nümbrecht, Germany) and a DNeasy Blood and Tissue Kit for DNA purification (Qiagen, Hilden, Germany). The filters were incubated by submersion in a mixed buffer ( $400 \mu \mathrm{L}$ buffer $\mathrm{AL}$ and $40 \mu \mathrm{L}$ Proteinase K; Qiagen) using a Salivette tube in a dry oven at $56^{\circ} \mathrm{C}$ for $30 \mathrm{~min}$. The tubes with filters were centrifuged at $5,000 \mathrm{~g}$ for $5 \mathrm{~min}$ at room temperature. Then, $220 \mu \mathrm{L}$ of TE (Tris-EDTA) buffer (pH: 8.0; $10 \mathrm{mM}$ Tris$\mathrm{HCl}$ and $1 \mathrm{mM} \mathrm{EDTA}$ ) was added to the filters, and tubes were centrifuged again at 5,000 $\mathrm{g}$ for 5 min. Buffer AL $(200 \mu \mathrm{L})$ and $100 \%$ ethanol $(600 \mu \mathrm{L})$ were then added to each filtrate and mixed by pipetting. The mixture was applied to a DNeasy Mini spin column and centrifuged at $6,000 \mathrm{~g}$ for $1 \mathrm{~min}$. This step was repeated until the mixture was completely processed. We followed the manufacturer's instructions for further steps, and eDNA was eluted from each sample solution with a final volume of $100 \mu \mathrm{L}$ Buffer AE.

The eDNA samples were then used for PCR-RFLP assay to detect DNA from the three target Japanese brown frog species based on Igawa et al (2015). This method utilizes speciesspecific restriction enzyme (SpeI and HphI) digestion sites in a partial nucleotide fragment of mitochondrial 16S rRNA amplified by PCR. However, the oligonucleotide primers used by Igawa et al. (2015) could amplify 16S rRNA fragments originating from other vertebrate species including humans which showed similar banding patterns. Therefore, we altered the primers to amplify a shorter region in which only the three target frog species have restriction sites. Specifically, we modified the method of Igawa et al (2015) by changing the primers: F96 5'GTCCAGCCTGCCCAGTGAYAAA -3' and R19 5'- GTTGAACAAACGAACCATTGGT -3'. These new primers were designed from an internal region of the previous study and amplify 
199

200

201

202

203

204

205

206

207

208

209

210

211

212

213

214

215

216

217

218

219

220

221

222

223

224

225

226

227

228

229

230

231

232

233

234

235

236

237

238

shorter fragments (R. japonica: $514-517$ bp, R. ornativentris: 516-517 bp and R. t. tagoi: 514-516 bp).

For higher efficiency of PCR amplification, we also modified the PCR protocol described by Igawa et al. (2015). In this study, PCR was conducted using KOD FX Neo (TOYOBO, Osaka, Japan). The $20 \mu \mathrm{L}$ total volume of reaction solution included $10 \mu \mathrm{L}$ of $2 \times$ PCR Buffer for KOD FX Neo, $2 \mu \mathrm{L}$ of $2 \mathrm{mM}$ dNTPs, 10 pmole of each primer, $3 \mu \mathrm{L}$ of eDNA solution and $0.4 \mathrm{U}$ of KOD FX Neo. Thermal cycling was performed using two-step PCR cycling: $95^{\circ} \mathrm{C}$ for 3 min followed by 35 cycles of $98^{\circ} \mathrm{C}$ for $10 \mathrm{~s}$ and $65^{\circ} \mathrm{C}$ for $30 \mathrm{~s}$. Following PCR amplification, independent digestion using two restrictions enzymes (SpeI and HphI) and electrophoresis were conducted in the same manner as Igawa et al. (2015). Then, $5 \mu 1$ of the original PCR product or $15 \mu l$ of each PCR product digested with the two restriction enzymes were electrophoresed on a $2 \%$ agarose gel for $30 \mathrm{~min}$ at $100 \mathrm{~V}$ and visualized. We performed the experiment several times to confirm the reproducibility.

For $R$. japonica, the amplified fragments are expected to have different digestion patterns between the western (including Hiroshima) and northern part (including Fukushima) of the Japanese mainland (Igawa et al., 2015). In all $R$. japonica populations, amplicons digested with SpeI result in 255 and 259 bp subfragments. However, after Hph1 digestion, R. japonica frogs from the western part have no subfragments, while those from the northern part have 301 and 220 bp subfragments (Fig. 2A). For $R$. ornativentris and $R$. t. tagoi, amplified fragments are commonly digested only with HphI, resulting in 231, 199 and 86 bp subfragments and 313 and 200 bp subfragments, respectively (Fig. 2A). To confirm the specificity of eDNA detection by our PCR-RFLP method, PCR products of sample no. 1, 10, 11, 14, 17, 19 and 20 were directly sequenced using the same primers and BigDye Terminator ver 3.1 (Life Technologies) after PEG precipitation. The obtained nucleotide sequences were annotated by NCBI blastn (https://blast.ncbi.nlm.nih.gov).

\section{qPCR assay}

To compare sensitivity between the methods, we conducted real-time quantitative PCR (qPCR) assay for samples from Fukushima to compare the sensitivity of eDNA detection for $R$. $t$. tagoi. At first, we defined regions to design the primers and TaqMan MGB probes for the qPCR assays for this species using PrimerExpress 3.0.1 (Thermo Fisher Scientific, Carlsbad, CA, USA), for amplification of 106 bp fragments: Rtag16SrRNA-F, 5'-

AGAAGGAACTCGGCAAACCTT-3'; Rtag16SrRNA-R, 5'-CCGCGGCCGTTGAAT-3'; Rtag16SrRNA-Pr, 5'-[FAM]-CCAGCCTGCCCAGTG-[NFQ]-[MGB]-3'. To prove that the primers did not amplify other sympatric frog species, we confirmed no amplification when the primers were applied to DNA extracted from $R$. japonica and $R$. ornativentris tissues. However, in addition to $R$. $t$. tagoi, three closely related species (i.e., $R$. sakuraii, $R$. t. okiensis and $R$. $t$. yakushimensis) were also detected in an in silico specificity screen, which was performed using Primer-BLAST, with the setting "nr" for database and "vertebrates" for organisms parameters (http://www.ncbi.nlm.nih.gov/tools/primer-blast/). Rana sakuraii may inhabit sympatrically with 
239 R. t. tagoi in our field survey. In contrast, Rana t. okiensis and R. t. yakushimensis never share

240 distribution with our target species because they are only found on Oki island in Shimane

241 Prefecture and the Yakushima islands in Kagoshima Prefecture, respectively. Thus, in this study,

242 the qPCR primers we developed are specific for $R$. t. tagoi/R. sakuraii.

243 Environmental DNA was quantified using a StepOnePlus ${ }^{\text {TM }}$ Real-Time PCR system (Life

244 Technologies, Carlsbad, CA, USA). Each TaqMan reaction contained $10 \mu \mathrm{L}$ of TaqMan ${ }^{\circledR}$

245 Environmental Master Mix 2.0 (Thermo Fisher Scientific), $1 \mu \mathrm{L}$ of the primer $(900 \mathrm{nM}) /$ probe

$246(125 \mathrm{nM}) \mathrm{mix}, 7 \mu \mathrm{L}$ of distilled water, and $2 \mu \mathrm{L}$ of eDNA extract. The PCR cycles were as

247 follows: $2 \mathrm{~min}$ at $50^{\circ} \mathrm{C}, 10 \mathrm{~min}$ at $95^{\circ} \mathrm{C}$, then 55 cycles of $15 \mathrm{~s}$ at $95^{\circ} \mathrm{C}$, and $60 \mathrm{~s}$ at $60^{\circ} \mathrm{C}$. In

248 order to produce standard DNA for the qPCR, a target amplicon was inserted into a pMD20-T

249 vector (Takara Bio, Shiga, Japan), and the vector was digested with BamHI. A dilution series of

250 the plasmid containing $1 \times 10^{1}$ to $1 \times 10^{4}$ copies was amplified as standards in duplicates in all

251 qPCR assays. The qPCR for each sample was performed in 8 wells, and the mean was used as

252 the concentration of eDNA (copies/L). If any of the 8 replicates of each sample yielded a

253 positive result, the sample was designated as containing $R$. t. tagoi/R. sakuraii eDNA. As a

254 negative control, each qPCR assay included 8 wells that contained no template ( $2 \mu \mathrm{L}$ of DNA-

255 free water) (i.e. NTC). In addition, using the primer-probe set of this species, qPCR amplicons

256 were sequenced directly from a positive PCR of the field samples $(\mathrm{N}=6)$ after treatment with

257 ExoSAP-IT (USB Corporation, Cleveland, OH, USA) or DNA fragment isolation with

258 FastGene $^{\mathrm{TM}}$ Gel/PCR Extraction Kit (NIPPON Genetics, Tokyo, Japan) following agarose gel

259 electrophoresis. Products were sequenced by a commercial sequencing service (Takara Bio,

260 Shiga, Japan) or by our experiment using ABI3130xl automated sequencer (Thermo Fisher

261 Scientific).

262 The range of qPCR efficiency across the entire study, calculated from the slope of

263 standard curves, was $91.937 \%$ to $94.716 \%$, and the $\mathrm{R}^{2}$ value for standard curve was 0.997 . Since

264 we could detect two copies of DNA in at least one of the three replicates, we defined the limit of

265 detection (LOD, the lowest concentration where all triplicate samples registered a Ct value) for

266 DNA from $R$. $t$. tagoi using qPCR assay as two copies.

267

268

269 Results

270 Water tank experiments

$271 \quad$ For the eDNA solutions from water tank experiments, we successfully amplified $16 \mathrm{~S}$

272 rRNA fragments from all samples, each showing a single band following gel electrophoresis

273 (Fig. 2B). The band patterns of digested fragments in each sample were completely matched with

274 the species that were held in each tank, except for the negative control tank without animals (Fig.

275 2B). Notably, for the samples from water tanks in which two species were cultured, we could

276 simultaneously amplify eDNA from both species whereby both band patterns specific to two

277 species were displayed.

278 
279

280

281

282

283

284

285

286

287

288

289

290

291

292

293

294

295

296

297

298

299

300

301

302

303

304

305

306

307

308

309

310

311

312

313

314

315

316

317

318

\section{Field survey and experiments}

In the survey in Fukushima (A), field observation of $R$. t. tagoi adults were recorded in 15 of 19 sites (Fig 1, Supp Table 1). Among the sites where $R$. $t$. tagoi adults were observed, adults of $R$. ornativentris were also observed sympatrically in 5 sites (Supp Table 1). However, we could not identify presence of either $R$. t. tagoi or $R$. ornativentris by PCR-RFLP analyses of eDNA from any of the samples from Fukushima (Supp Fig 1). Subsequently, we applied the more sensitive $R$. t. tagoi/R. sakuraii-specific qPCR which could still only confirm presence of R. t. tagoi in five of the 15 sites with field observations of adults of the species (Fig 1, Supp Table 1). Our direct sequences of the qPCR amplicons (106 bp product size) were almost identical to haplotypes of $R$. $t$. tagoi deposited in DDBJ. In addition, no amplification was observed in any control samples (i.e. filtering controls and NTC).

We could obtain clear sequence data of PCR products from site no. 1 and 21, while the others showed mixed chromatograms that could not be further interpreted. In Fukushima site no. 1, we also reported field observation of Bufo japonicus tadpoles, a species outside of our target species. PCR amplification of eDNA from the same site resulted in an approximately $600 \mathrm{bp}$ fragment; subsequent sequencing and BLAST search confirmed a match of nucleotide sequence with partial sequence of $B$. japonicus $(=B . j$. formosus) 16S rRNA [AB159565, a haplotype from Atsumi, Yamagata Pref. (Igawa et al., 2006)].

From field experiment (B) and (C), field observation of $R$. japonica tadpoles was recorded for three sites (no. 21, 22, and 27; Fig 1, Supp Table 1). This was consistent with our PCR-RFLP which showed $R$. japonica-specific banding patterns following SpeI restriction digestion in the same samples (Fig. 2C). We also confirmed the sequence from site no. 21 had BLAST identity with partial sequence of 16s rRNA from $R$. japonica [LC014155, a haplotype from Hiroshima University (Igawa et al., 2015)]. In addition, PCR-RFLP analyses of site no. 28 supports the presence of $R$. japonica, despite no field observation of tadpoles at this small drainage site. We also confirmed amplification of a fragment that may be amplified from nontarget species in site no. 21, 22, 27 and 28. These fragments were digested with HphI and showed weak bands around $400 \mathrm{bp}$ and/or $300 \mathrm{bp}$ that were different from the target species.

\section{Discussion}

As shown in our water tank experiment, eDNA of Rana species was successfully amplified and we could even simultaneously identify each pair of the three species using PCRRFLP method. Following success of this newly developed protocol, we applied it to a variety of field collected water samples.

In field experiment (A) at Fukushima, despite frogs being identified in 15 of 19 sites by field observation, we could not detect eDNA of $R$. $t$. tagoi or $R$. ornativentris using our PCRRFLP method. Even when we applied the more sensitive qPCR approach for detecting $R$. $t$. tagoi, this method only confirmed presence of the species in five of the sites. There are differences in detection sensitivity across PCR-RFLP and qPCR methods: qPCR is rather 
319

320

321

322

323

324

325

326

327

328

329

330

331

332

333

334

335

336

337

338

339

340

341

342

343

344

345

346

347

348

349

350

351

352

353

354

355

356

357

358

sensitive compared to conventional PCR in this case.. In addition, the more expensive approaches like digital PCR and NGS of eDNA could prove to be more sensitive, despite the hurdles associated with cost.

The lack of detection by PCR-RFLP and limited sensitivity by qPCR could be attributed to the water system of the environment. Such factors like water volume, water discharge and flow rate could markedly impact the concentration of eDNA. It seems that flowing waters of lotic habitats, such as in rivers or streams, could have lower eDNA concentrations due to dilution from fresh water upstream. In particular, while only $500 \mathrm{~mL}$ water samples were collected in this study, previous studies extracted eDNA from more than $1 \mathrm{~L}$ of water (Pilliod et al., 2013; Baldigo et al., 2017). Such difference in water sampling volume might be associated with the lack/detection of eDNA. In the future, increasing the volume of water collection might improve eDNA detection power. In addition, $R$. $t$. tagoi adults and their spawned tadpoles utilize small torrents or subsoil water systems only in the breeding season. According to Maeda and Matsu (1999), R. t. tagoi breed during late June in colder regions of Japan like Fukushima; our sample sessions occurred at both before and after this breeding period and thus could result in low level of eDNA in the water. Overall, the detection of Rana species from river water samples could potentially be challenging compared to static water samples, and we need to select the appropriate time of sampling considering ecology and behavior of the target species.

Nevertheless, if future optimization can overcome these limitations, there is potential to apply such approaches to effectively monitor distribution of frogs in regenerating habitats in vicinity to the FNPP.

We also applied PCR-RFLP to static water samples collected in the field. From the eight sites in field experiments (B) and (C), PCR-RFLP could successfully detect eDNA in all three sites where $R$. japonica tadpoles were visually observed. An additional site, site no. 28, had no tadpoles observed in field study, but showed some evidence of presence from PCR-RFLP analyses. This site is a small drainage site with a small volume and water flowing upstream from a paddy field. Thus, it seems possible that PCR-RFLP can detect presence of frogs that may inhabit upstream or in close proximity, even in the absence of visual confirmation. When we compare this site of flowing water to that of sites in Fukushima, the water volumes and flow rate are much smaller and slower, respectively.

\section{Conclusion}

We have successfully developed a cost-effective method for detecting three common Rana frog species from Japan. This approach works well in field-collected samples that originate from static or slow-flowing water systems. However, our approach cannot be applicable to lotic water systems. Further exploration of alternative cost-effective methods are required, and could include collection of larger volumes of water and experimental assessment of the impact of various factors like water volume and flow rate on eDNA concentration. Nevertheless, this method has potential to be used to monitor frog populations that are facing decline due to 
359

360

361

362

363

364

365

366

367

368

369

370

371

372

373

374

375

376

377

378

379

380

381

382

383

384

385

386

387

388

389

390

391

392

393

394

395

396

397

398

399

400

urbanization, especially $R$. japonica which inhabits static water bodies like rice paddy fields. Our approach also has potential to be adapted and optimized for detection of other amphibian species from field sites.

\section{Acknowledgements}

The present study was financially supported by a Scientific Research Grant from the Kurita Water, and Environment Foundation Grant and by the Environment Research and Technology Development Fund of the Ministry of the Environment (4RF-1302), and Environmental Restoration and Conservation Agency (4-1602 and 4-1503), Japan. We also thank the Department of Molecular and Functional Genomics, Interdisciplinary Center for Science Research, Organization for Research and Academic Information, Shimane University, for providing the DNA laboratory facilities. The authors thank the faculty of Life and Environmental Science in Shimane University, Japan for providing financial support towards publishing this study. All methods adhered to the laws of Japan.

\section{References}

Baldigo BP., Sporn LA., George SD., Ball JA. 2017. Efficacy of Environmental DNA to Detect and Quantify Brook Trout Populations in Headwater Streams of the Adirondack Mountains, New York. Transactions of the American Fisheries Society 146:99-111. DOI: 10.1080/00028487.2016.1243578.

Biggs J., Ewald N., Valentini A., Gaboriaud C., Dejean T., Griffiths RA., Foster J., Wilkinson JW., Arnell A., Brotherton P., Williams P., Dunn F. 2015. Using eDNA to develop a national citizen science-based monitoring programme for the great crested newt (Triturus cristatus). Biological Conservation 183:19-28. DOI: 10.1016/j.biocon.2014.11.029.

Buxton AS., Groombridge JJ., Zakaria NB., Griffiths RA. 2017. Seasonal variation in environmental DNA in relation to population size and environmental factors. Scientific Reports 7:1-9. DOI: 10.1038/srep46294.

Clusa L., Ardura A., Fernández S., Roca AA., García-Vázquez E. 2017. An extremely sensitive nested PCR-RFLP mitochondrial marker for detection and identification of salmonids in eDNA from water samples. PeerJ 5:e3045. DOI: 10.7717/peerj.3045.

Goldberg CS., Strickler KM., Pilliod DS. 2015. Moving environmental DNA methods from concept to practice for monitoring aquatic macroorganisms. Biological Conservation 183:13. DOI: 10.1016/j.biocon.2014.11.040.

Gosner KL. 1960. A Simplified Table for Staging Anuran Embryos Larvae with Notes on Identification. Herpetologica 16:183-190.

Igawa T., Komaki S., Takahara T., Sumida M. 2015. Development and Validation of PCR-RFLP Assay to Identify Three Japanese Brown Frogs of the True Frog Genus Rana. Current Herpetology 34:89-94. DOI: 10.5358/hsj.34.89.

Igawa T., Kurabayashi A., Nishioka M., Sumida M. 2006. Molecular phylogenetic relationship of toads distributed in the Far East and Europe inferred from the nucleotide sequences of 
401

402

403

404

405

406

407

408

409

410

411

412

413

414

415

416

417

418

419

420

421

422

423

424

425

426

427

428

429

430

431

432

433

434

435

436

437

438

439

440

441

442

443

444

445

446

mitochondrial DNA genes. Molecular phylogenetics and evolution 38:250-60. DOI: 10.1016/j.ympev.2005.09.003.

Iwai N., Yasumiba K., Takahara T. 2019. Efficacy of environmental DNA to detect and quantify stream tadpoles of Odorrana splendida. Royal Society Open Science 6:181798. DOI: 10.1098/rsos.181798.

Katano I., Harada K., Doi H., Souma R., Minamoto T. 2017. Environmental DNA method for estimating salamander distribution in headwater streams, and a comparison of water sampling methods. PLOS ONE 12:e0176541. DOI: 10.1371/journal.pone.0176541.

Kitagawa Y. 2002. Regeneration of Satoyama Landscapes. In: Takeuchi K, Brown RD, Washitani I, Tsunekawa A, Yokohari M eds. Satoyama: The Traditional Rural Landscape of Japan. Tokyo: Springer-Verlag, 129-142.

Kuramoto M., Ishikawa H. 2000. Breeding ecology of brown frogs in Yamada park, Kitakyushu, Japan. Bull Herpetol Soc Jpn 2000:7-18.

Maeda N., Matsui M. 1999. Frogs and Toads of Japan. Tokyo: Bunichi Sogo Shuppan.

Natural Environment Division, Bureau of Environment TMG. 2010. 2010 Red List of Threatened Species in Tokyo: 23-ward and Tama area version. Tokyo: Natural Environment Division, Bureau of Environment, Tokyo Metropolitan Government.

Natural Environment Division, Depertment of Environmental Affairs FPG. 2014. Endangered Species in Fukuoka: Red Data Book Fukuoka 2014. Fukuoka: Natural Environment Division, Depertment of Environmental Affairs, Fukuoka Prefectural Government,.

Olson ZH., Briggler JT., Williams RN. 2013. An eDNA approach to detect eastern hellbenders (Cryptobranchus a. alleganiensis) using samples of water. Wildlife Research 39:629. DOI: 10.1071/WR12114.

Osawa S., Katsuno T. 2001. Dispersal of Brown Frogs Rana japonica. Current Herpetology 20:1-10.

Pilliod DS., Goldberg CS., Arkle RS., Waits LP. 2013. Estimating occupancy and abundance of stream amphibians using environmental DNA from filtered water samples. Canadian Journal of Fisheries and Aquatic Sciences 70:1123-1130. DOI: 10.1139/cjfas-2013-0047.

Rees HC., Maddison BC., Middleditch DJ., Patmore JRM., Gough KC. 2014. REVIEW: The detection of aquatic animal species using environmental DNA - a review of eDNA as a survey tool in ecology. Journal of Applied Ecology 51:1450-1459. DOI: 10.1111/13652664.12306.

Tahara Y. 1974. Table of the Normal Developmental Stages of the Frog, Rana japonica. Memoirs of Osaka Kyoiku University. III, Natural science and applied science 23:33-53.

Takahara T., Iwai N., Yasumiba K., Igawa T. An eDNA method for detection of three endangered frog species in Japan by analyzing seasonal shift in detection efficiency and comparison with acoustic survey. Freshwater Science In press.

Takahara T., Minamoto T., Doi H. 2013. Using Environmental DNA to Estimate the Distribution of an Invasive Fish Species in Ponds. PLoS ONE 8. DOI: 10.1371/journal.pone.0056584.

Thomsen PF., Willerslev E. 2015. Environmental DNA - An emerging tool in conservation for monitoring past and present biodiversity. Biological Conservation 183:4-18. DOI: 10.1016/j.biocon.2014.11.019.

Uchii K., Doi H., Minamoto T. 2015. A novel environmental DNA approach to quantify the cryptic invasion of non-native genotypes. Molecular Ecology Resources:n/a-n/a. DOI: 10.1111/1755-0998.12460. 
Figure 1

Sampling localities of field experiments.

Sampling localities of field experiments: (A) regional sampling in Fukushima, (B) local area sampling in Hiroshima University, (C) microhabitat level sampling in Etajima Island. Results of field observation, GRCR and PCR-RFLP detection were indicated, respectively $(\mathrm{X} / \mathrm{X} / \mathrm{X})$. +: detected. -: not detected. NA: qPCR not conducted.
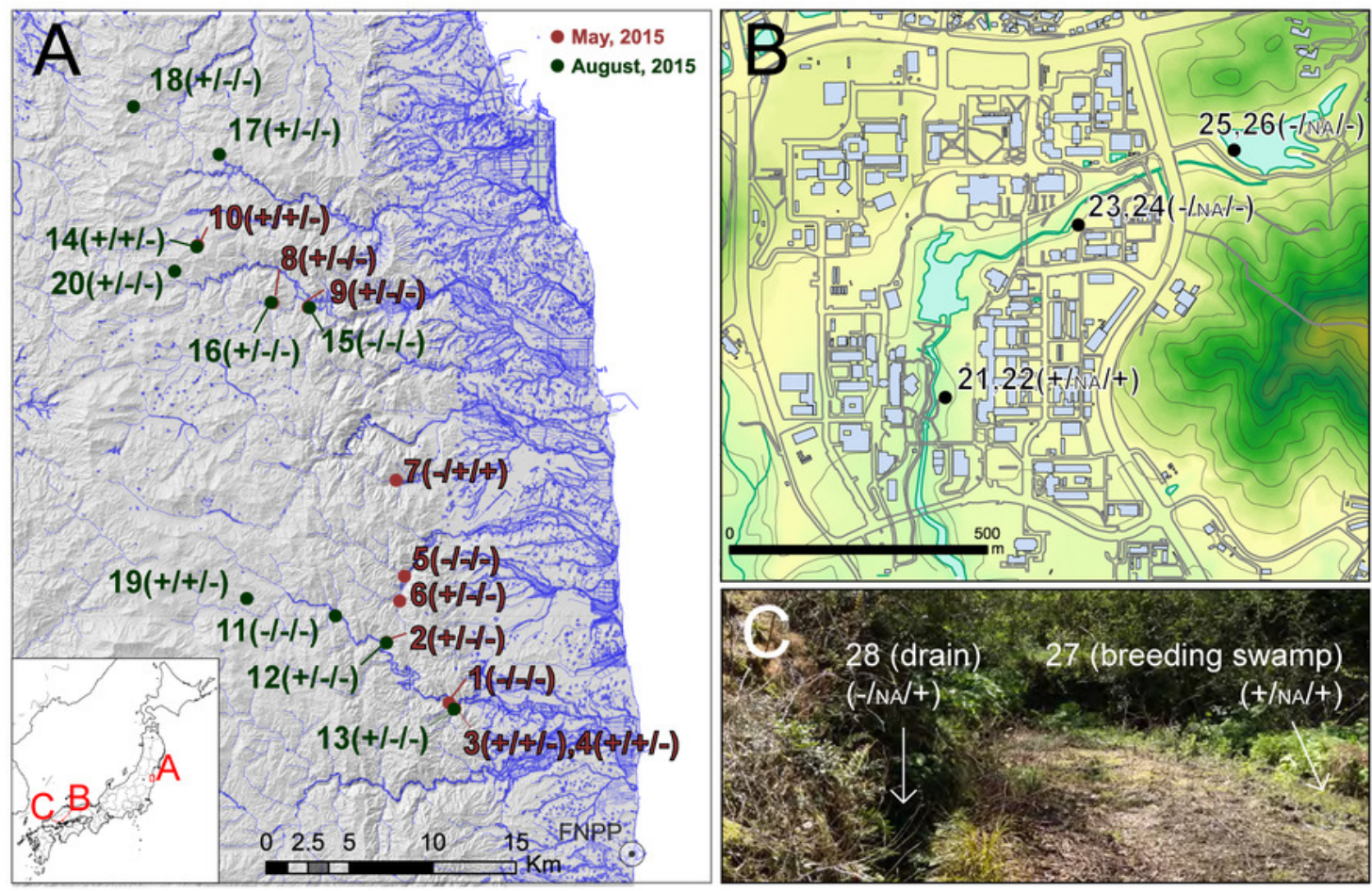
Figure 2

PCR-RFLP and gel electrophoresis of water tank experiment (A) and field experiment (B). 
A

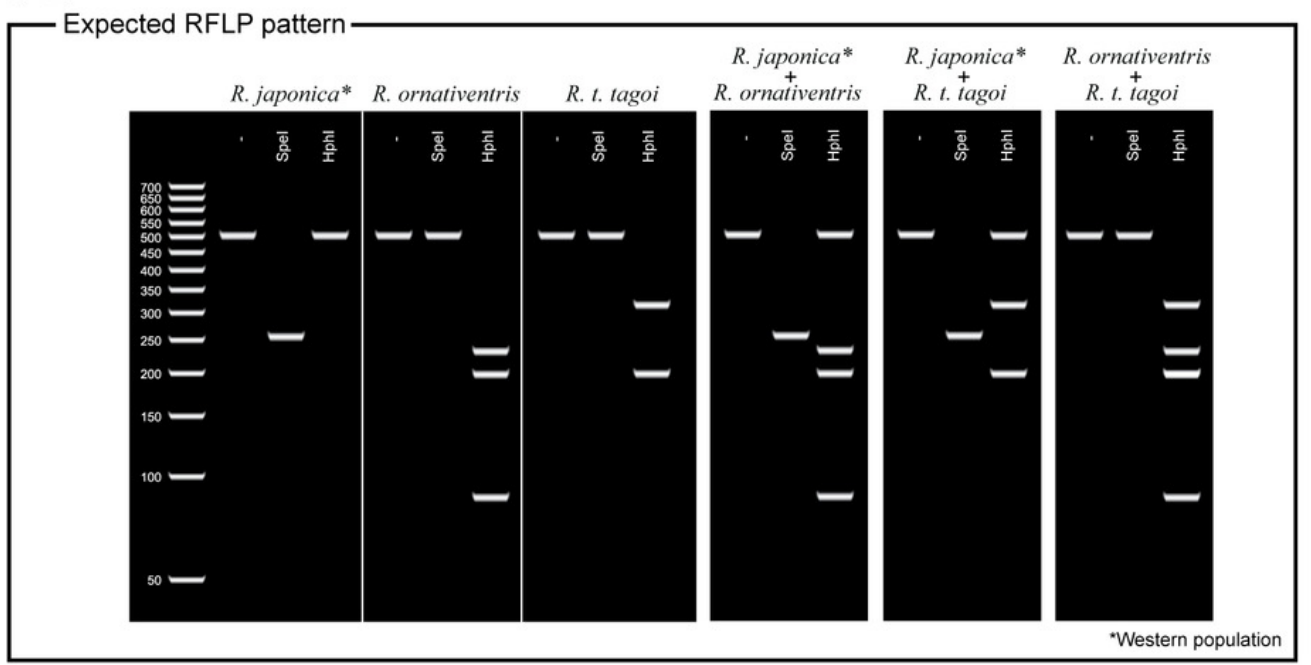

\section{B}

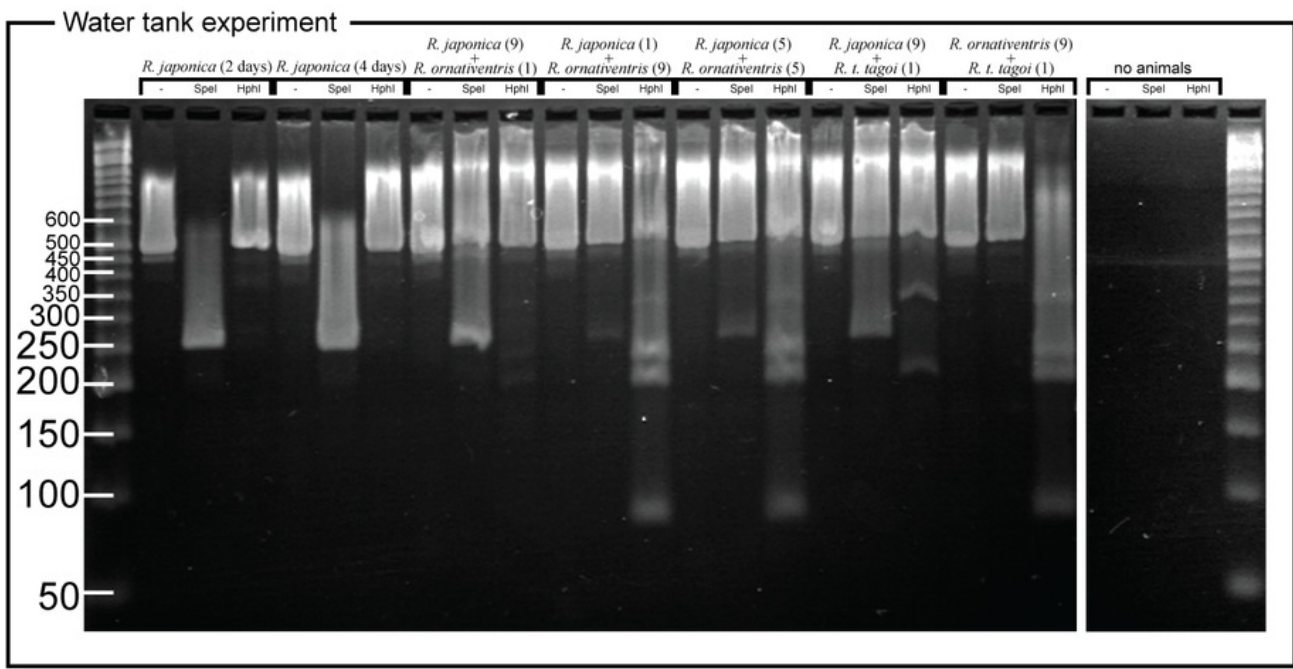

\section{C}

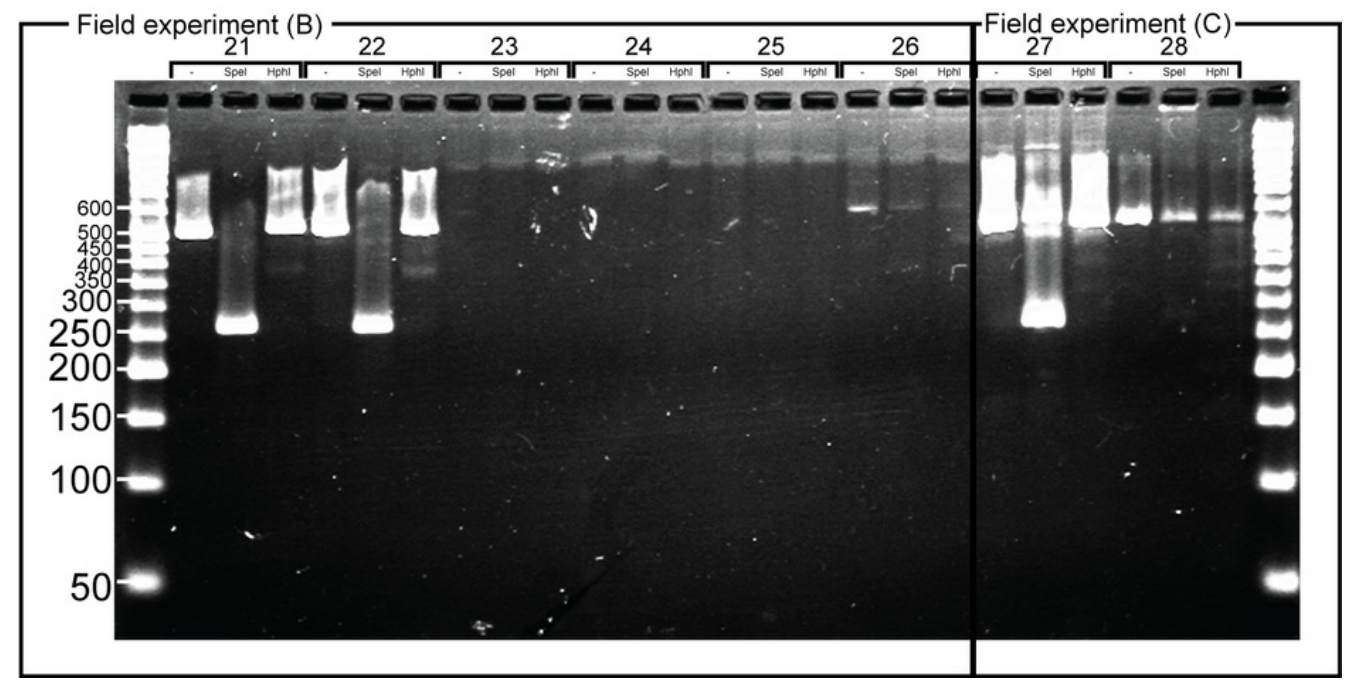

Peer) reviewing PDF | (2019:03:35641:1:1:NEW 4 Jul 2019) 